\title{
Myc confers androgen-independent prostate cancer cell growth
}

\author{
David Bernard, ${ }^{1}$ Albin Pourtier-Manzanedo, ${ }^{2}$ Jesús Gil, ${ }^{1}$ and David H. Beach ${ }^{1}$ \\ ${ }^{1}$ Wolfson Institute for Biomedical Research, University College London, London, United Kingdom \\ ${ }^{2}$ Sciences Naturelles 3, Université des Sciences et Techniques de Lille, Villeneuve D’Ascq, France
}

\begin{abstract}
Prostate cancer is one of the most diagnosed and mortal cancers in western countries. A major clinical problem is the development of androgen-independent prostate cancer (AIPC) during antihormonal treatment. The molecular mechanisms underlying the change from androgen dependence to independence of these tumors are poorly understood and represent a challenge to develop new therapies. Based on genetic data showing amplification of the c-myc gene in AIPC, we studied the ability of c-myc to confer AIPC cell growth. Human androgen-dependent prostate cancer cells overexpressing c-myc grew independently of androgens and presented tumorigenic properties in androgen-depleted conditions. Analysis of signalling pathways by pharmacological inhibitors of the androgen receptor (AR) or by RNA interference directed against AR or c-myc showed that c-myc acted downstream of AR through multiple growth effectors. Thus c-myc is required for androgen-dependent growth and following ectopic expression can induce androgen-independent growth. Moreover, RNA interference directed against c-myc showed that growth of human AIPC cells, AR-positive or -negative, required c-myc expression. Furthermore, we showed that c-myc-overexpressing cells retain a functional p53 pathway and thus respond to etoposide.
\end{abstract}

J. Clin. Invest. 112:1724-1731 (2003). doi:10.1172/JCI200319035.

\section{Introduction}

Apart from skin cancer, prostate cancer is the most frequently diagnosed cancer and the second leading cause of death as a result of cancer for men in the United States $(1,2)$. During its initial stages, prostate tumor progression is dependent of AR signalling triggered by dihydrotestosterone (DHT), a metabolically active androgen subproduct. Because of this, besides prostatectomy and radiotherapy in localized prostate cancer, the main treatment for advanced prostate cancer is androgen ablation by surgery or chemical castration. Despite the general success of antiandrogen therapy, a negative outcome of this treatment is the appearance of androgen-refractory tumors, with an eventual fatal prognosis. Thus, finding an effective treatment for androgen-independent prostate cancer (AIPC) remains an important challenge. Consequently, understanding

Received for publication May 27, 2003, and accepted in revised form September 30, 2003.

Address correspondence to: David H. Beach, Wolfson Institute for Biomedical Research, University College London, Gower Street, London WC1E 6BT, United Kindom.

Phone: 0032-2555-6016; Fax: 0032-2555-6257;

E-mail: dhbeach@btinternet.com.

David Bernard's present address is: Free University of Brussels Laboratory of Molecular Virology Faculty of Medicine, Brussels, Belgium.

Conflict of interest: The authors have declared that no conflict of interest exists.

Nonstandard abbreviations used: dihydrotestosterone (DHT); androgen-independent prostate cancer (AIPC); androgen receptor (AR); p53 dominant negative (p53DN); pRetroSuper (pRS); prostrate specific antigen (PSA); prostrate specific membrane antigen (PSMA). the molecular mechanisms of the transition of prostate cancers from androgen dependence to independence should be the first step in this process $(1,2)$.

Two main mechanisms have been proposed for explaining the development of AIPC. The first is based on an increase of androgen receptor (AR) signalling during the development of androgen-independent tumors. This increased signalling may be caused by AR mutations allowing the receptor to be activated by new ligands, by AR amplifications rendering AR signalling sensitive to low concentrations of DHT, or by AR signalling induction by a tyrosine kinase receptor, such as Her/2neu, in a ligand-independent manner $(2,3)$. It has been widely demonstrated that under antihormonal treatment there is pressure to select for a mutant $A R$ gene. These AR mutants have new steroid-binding characteristics and thus, depending on the mutation, can be activated by glucocorticoids or flutamide $(4,5)$. Except for $A R$ mutations and despite strong experimental evidence for all these mechanisms, the other hypotheses explaining AR signalling increases are subject to discrepancies (6-9). The second mechanism for AIPC is based on the induction of a positive growth signal independent of the AR that can overcome the growth inhibition imposed by antiandrogen therapies, thus establishing a bypass pathway (2).

A useful approach to better understand the genetic events underlying prostate tumor development is the genetic analysis of DNA amplifications. Using comparative genomic hybridization, a short region of chromosome $8 \mathrm{q}$ containing the c-myc gene has been delimited as a region commonly amplified during the appearance of AIPC $(10,11)$. Fluorescence in situ hybridization studies have also confirmed the specific amplification 
of the c-myc gene in up to $72 \%$ of AIPCs (10), and more interestingly, a significant increase of c-myc amplification has been observed as a consequence of antiandrogen treatment (12). The c-myc gene is a strong positive regulator of cell growth, and mutations in this gene are among the most common genetic lesions found in a wide variety of human cancers (13). In order to define the role of $\mathrm{c}$-myc in the appearance of AIPC, we have examined the effects of $c$-myc expression in a human androgen-sensitive prostate tumor cell line.

\section{Methods}

Cell culture and retroviral infection. LNCaP, PC-3, and DU145 cells (American Type Culture Collection, Rockville, Maryland, USA) were cultured in RPMI 1640 (Invitrogen Corp., San Diego, California, USA) supplemented with 10\% FCS (Sigma-Aldrich, St. Louis, Missouri, USA) and glutamine (Invitrogen Corp.). 22Rv1 cells (American Type Culture Collection) were cultured in a mixture of 40\% RPMI 1640, 40\% DMEM (Invitrogen Corp.), 20\% FCS, and glutamine. LAPC-4 cells (3) were cultured in Iscove media (Invitrogen Corp.) supplemented with 15\% FCS, glutamine, and 10 nM R1881 (PE Biosystems, Foster City, California, USA). The packaging cell line 293GP (Clontech Laboratories Inc., Palo Alto, California, USA) was grown in DMEM supplemented with 10\% FCS. Retrovirus production and infection of target cells was performed as described previously (14). The retroviral vectors used were: pHygroMarXII/c-myc, pHygroMarXIV/GFP, pBabepuroE7, pWZLblast/p53 $3^{175 \mathrm{H}}$ (p53DN) LZRS/Skp2, pBabepurohTERT, pLPCpurocyclinD1, and pWZLhygroCDK4. The target sequence used to silence c-myc was 5'-GAGGCGAACACACAACGTC$3^{\prime}$, and for AR was 5'-CAACCAGCCCGACTCCTTT-3'. These sequences were inserted in the pRetroSuper (pRS) retroviral vector (OligoEngine; DNAengine Inc., Seattle, Washington, USA) according to the manufacturer's recommendations to form $\mathrm{pRS} / \mathrm{myc}$ and $\mathrm{pRS} / \mathrm{AR}$. The infected cells were selected by $500 \mathrm{ng} / \mathrm{ml}$ puromycin, $100 \mu \mathrm{g} / \mathrm{ml} \mathrm{G} 418,50 \mu \mathrm{g} / \mathrm{ml}$ hygromycin, or $100 \mu \mathrm{g} / \mathrm{ml}$ blasticidin as required.

Growth curves and colony-formation assays. For the growth curves, 8,000 cells were seeded per well in 24-well plates. After 1 day, the cells were treated with bicalutamide at 10 $\mu \mathrm{g} / \mathrm{ml}$; this treatment was repeated every 2 days. Every 4 days, cells were washed in PBS, fixed in $0.5 \%$ glutaraldehyde (Sigma-Aldrich), and stained with $0.1 \%$ crystal violet (Sigma-Aldrich). Then crystal violet was dissolved in acetic acid and the relative cell number was determined by absorbance reading at $595 \mathrm{~nm}$. For the colony-formation assays, 250,000 LNCaP cells or 1,000,000 LAPC-4 cells were seeded in $10-\mathrm{cm}$ dishes. The bicalutamide treatment was performed as described above for the LNCaP cells. For LAPC-4 cells, antiandrogen treatment was started 5 days after seeding by adding bicalutamide at $2.5 \mu \mathrm{g} / \mathrm{ml}$ in media without R1881. Next, the cells were stained with crystal violet as described above.

Soft agar assays. Ten thousand cells were resuspended with $3 \mathrm{ml}$ of $0.35 \%$ low-melting-point agarose (Invitro- gen Corp.) in RPMI 1640 containing 10\% FCS or 8\% Charcoal/dextran treated FBS (CDS) (HyClone Laboratories, Logan, Utah, USA), $2 \%$ FCS, and $10 \mu \mathrm{g} / \mathrm{ml}$ bicalutamide. This upper layer was seeded into 6-well plates coated with $0.7 \%$ low-melting-point agarose in RPMI 1640 with $10 \%$ FCS, or in 8\% CDS, 2\% FCS, and $10 \mu \mathrm{g} / \mathrm{ml}$ bicalutamide. Every 3 days, $1 \mathrm{ml}$ of fresh media containing either $10 \%$ FCS or $8 \%$ CDS, $2 \%$ FCS, and $10 \mu \mathrm{g} / \mathrm{ml}$ bicalutamide was added. The number of foci was counted after 2 weeks.

Cell division analysis. After retroviral transduction, cells were selected for 5 days. Cells were trypsinized and stained with CFDA SE dye (Molecular Probes Inc., Eugene, Oregon, USA) according to the manufacturer's recommendations. After staining, 300,000 cells per $10-\mathrm{cm}$ dish were seeded in the presence of bicalutamide. Cell division was analyzed by flow cytometry after 1 day or 9 days.

DNA content analysis. After infection and selection, 500,000 cells were seeded in $10-\mathrm{cm}$ dishes. After 1 day, the cells were treated with bicalutamide at $10 \mu \mathrm{g} / \mathrm{ml}$; this treatment was repeated every 2 days. On day 12 of treatment, the cells were fixed in ice-cold $70 \%$ ethanol, washed in PBS, and then treated with $10 \mu \mathrm{g} / \mathrm{ml}$ RNase A for 30 minutes at $37^{\circ} \mathrm{C}$. Propidium iodide (Sigma-Aldrich) at $10 \mu \mathrm{g} / \mathrm{ml}$ was added to the samples prior to analysis on a FACSCalibur flow cytometer (Becton, Dickinson and Co., Franklin Lakes, New Jersey, USA).

Western blot analysis. Western blots were carried out using whole cell extracts obtained using RIPA buffer, separated on 8-12.5\% SDS-PAGE gels, and transferred to nitrocellulose membranes. Membranes were incubated with the following primary antibodies: anti-c-myc (sc764; Santa Cruz Biotechnology Inc., Santa Cruz, California, USA), anti-p16 (C-20; Santa Cruz Biotechnology Inc.), anti-rentinoblastoma protein (anti-RB) (554136; Pharmingen, San Diego, California, USA), anti-E2F1 (sc193; Santa Cruz Biotechnology Inc.), anti-cyclin A (sc596; Santa Cruz Biotechnology Inc.), anti-p27, anti-Skp2 (sc-7164; Santa Cruz Biotechnology Inc.), anti-cyclin D1, anti-CDK4 (sc-260; Santa Cruz Biotechnology Inc.), anti-ornithine decarboxylase (O1136; Sigma-Aldrich), anti-hTERT (sc-7212; Santa Cruz Biotechnology Inc.), anti-prostrate specific antigen (anti-PSA) (A0562; Dako Corp., Carpinteria, California, USA), anti-AR (554225; Pharmingen), anti- $\beta$-actin (A5316; Sigma-Aldrich), antip53 (sc-126; Santa Cruz Biotechnology Inc.), anti-p21 (sc-397; Santa Cruz Biotechnology Inc.), anti-GADD45 (sc-797; Santa Cruz Biotechnology Inc.), anti-prostrate specific membrane antigen (anti-PSMA) (sc-10269; Santa Cruz Biotechnology Inc.), and anti-neuron-specific enolase (M0873; Dako Corp.). The corresponding peroxidase-labelled secondary antibody (Santa Cruz Biotechnology Inc.) was detected using ECL Western blotting reagents (Amersham Biosciences, Piscataway, New Jersey, USA).

RT-PCR analysis. Cells were homogenized in Trizol (Invitrogen Corp.), and total RNA was isolated according to the manufacturer's recommendations. cDNA's 
were synthesized using the First Strand cDNA Synthesis Kit (Roche Diagnostics Corp., Indianapolis, Indiana, USA) and amplified in a final volume of $50 \mu \mathrm{l}$ containing $150 \mu \mathrm{M}$ dNTP, $2 \mathrm{mM} \mathrm{MgCl}_{2}, 1$ unit of Taq gold polymerase (Applied Biosystems, Foster City, California, USA), and each primer at $1 \mu \mathrm{M}$. Primers used were as follows: c-myc forward, CGACGCGGGGAGGCTATTCTGC; c-myc reverse, CCCGCCACCGCCGTCGTTGTCT; and $\beta$-actin, as described elsewhere (15). An initial denaturing step of 5 minutes at $95^{\circ} \mathrm{C}$ was followed by $25-30$ amplification cycles at $94^{\circ} \mathrm{C}$ for 1 minute, $63^{\circ} \mathrm{C}$ (c-myc) or $55^{\circ} \mathrm{C}\left(\beta\right.$-actin) for 1 minute, and $72^{\circ} \mathrm{C}$ for 1 minute.

\section{Results}

To study the functional effect of c-myc amplification observed in AIPC on prostate cancer cell growth during antihormonal treatment, we used LNCaP cells treated with bicalutamide, an AR antagonist, as a model. As expected, AR inhibition induced growth arrest of LNCaP control cells and $\mathrm{LNCaP}$ cells infected with a retroviral vector encoding GFP (Figure 1a). By contrast, although the growth of $\mathrm{LNCaP} /$ myc cells treated with bicalutamide was delayed compared with that of untreated $\mathrm{LNCaP} /$ myc cells, the treated cells continued to proliferate (Figure 1a). To further confirm that c-myc confers the ability to grow without androgen stimulation, we tested the capacity of these cells to form colonies when seeded at low density in the presence or absence of bicalutamide. Under these conditions, LNCaP control cells failed to form any colonies after 15 days of treatment (Figure 1b). By contrast, numerous colonies appeared in c-myc-expressing cells (Figure 1b). Next, we compared the cell cycle profile of control or c-myc-expressing cells treated with bicalutamide. The DNA profiles showed that only $2 \%$ of control cells were in S phase compared with $11 \%$ in c-mycexpressing cells, and $78 \%$ of control cells were in G1 phase versus $67 \%$ in c-myc-expressing cells (Figure 1c). These data indicate that c-myc overcame the blockage to cell cycle progression induced by AR inhibition.

To examine whether c-myc expression is sufficient to confer androgen-independent growth in LNCaP cells, a cell-by-cell division analysis during AR antagonist treatment was performed after retroviral transduction of c-myc. To this end, cells were stained with CFDA SE dye.

a

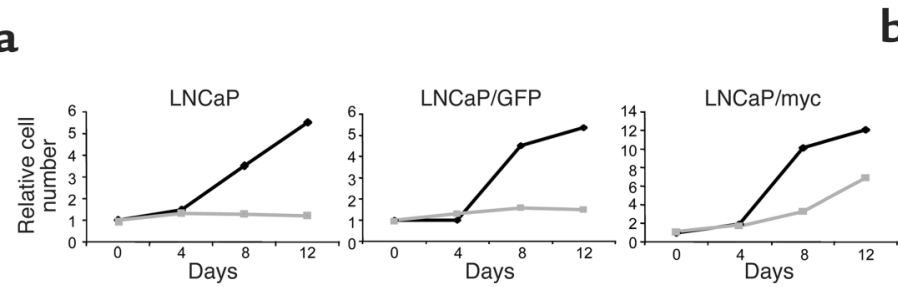

b

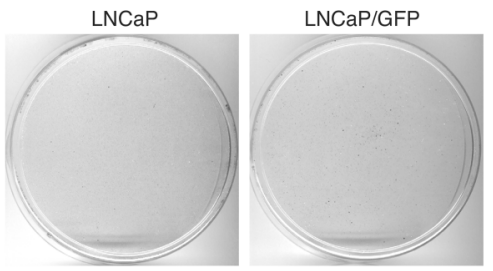

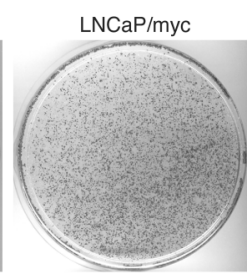

C
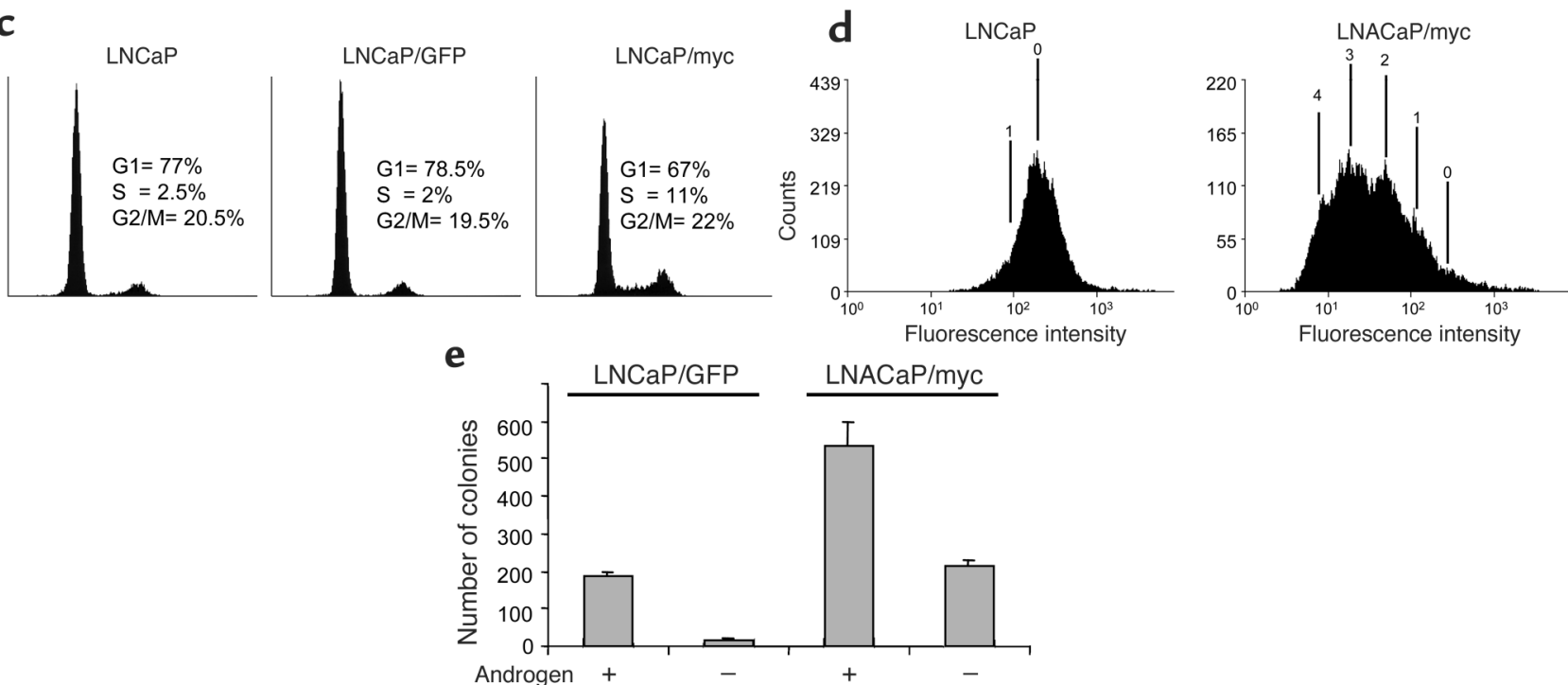

Figure 1

c-myc expression renders LNCaP cells androgen-independent. LNCaP cells were infected with GFP or c-myc retroviral vectors and selected with hygromycin. One day after seeding, bicalutamide treatment was started and renewed every 2 days. (a) Growth curve assays. Cells were seeded in triplicate in 24-well plates. At 4-day intervals, the cell number was analyzed. The black lines represent untreated cells, and the gray lines show bicalutamide-treated cells. (b) Colony-formation assays. Cells $(200,000)$ were seeded onto plates, and after 15 days of treatment with bicalutamide, the cells were stained using crystal violet. (c) DNA content analysis. After 11 days of bicalutamide treatment, cells were fixed, stained using propidium iodide, and analyzed. (d) Cell division analysis. After infection and selection, the cells were stained with CFDA SE dye and seeded in the presence of bicalutamide. Flow cytometry analysis on 10,000 cells was performed at day 9 and the number of cell divisions is presented. (e) Soft agar assays. Cells were plated in soft agar media containing or not containing androgen. Analysis of the appearance of the foci was performed after 2 weeks. 


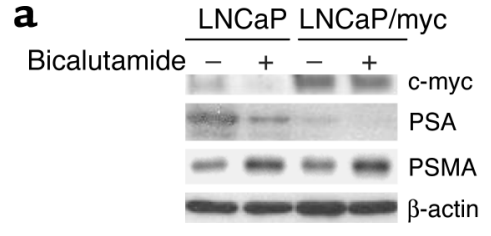

b
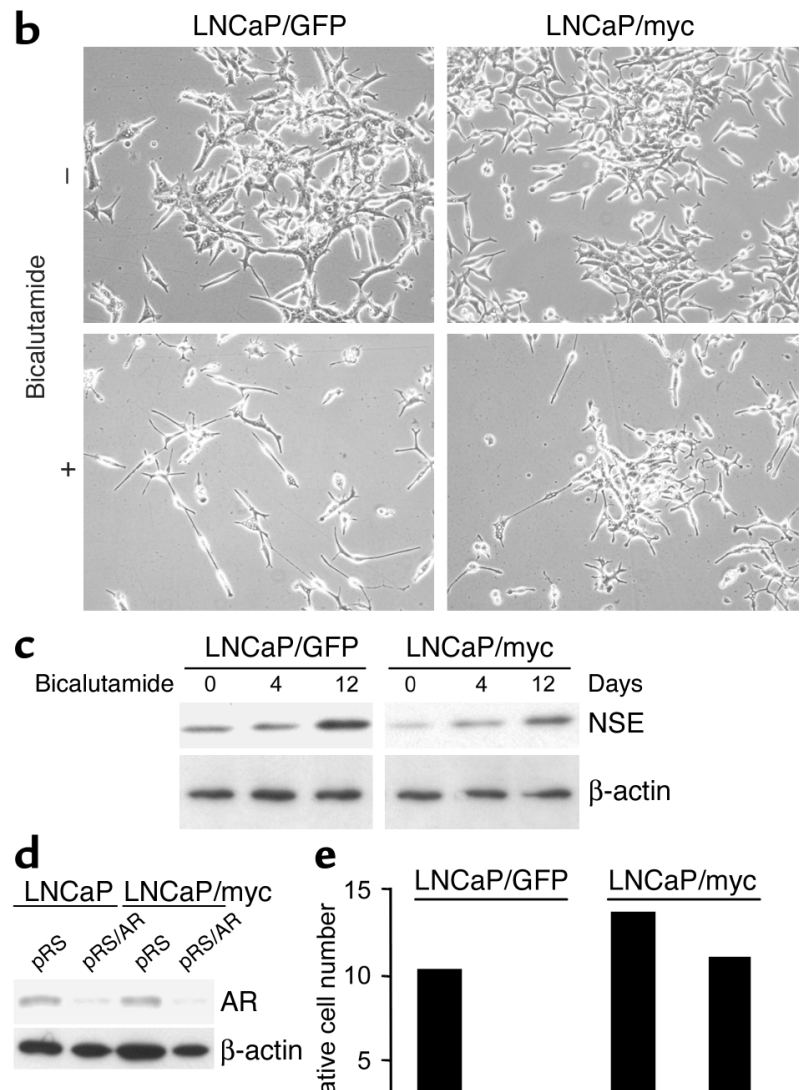

$\beta$-actin

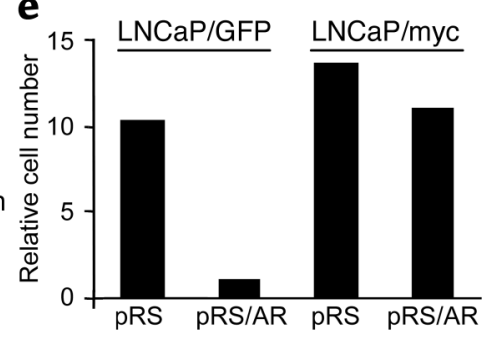

Figure 2

c-myc does not act through AR signalling. (a) c-myc does not induce PSA expression. Cells were treated with or without bicalutamide during 3 days. Cell extracts were resolved on SDS-PAGE gel, transferred, and analyzed for c-myc, PSA, and PSMA expression. $\beta$-Actin was used as loading control. (b) Cells were cultured with or without bicalutamide for 12 days after seeding. Representative pictures are presented. (c) Cellular extracts were prepared before bicalutamide treatment or after 4 and 12 days of treatment, resolved by SDS-PAGE, and analyzed by immunoblotting against neuron-specific enolase (NSE) and $\beta$-actin as a loading control. (d) Silencing of AR expression. LNCaP cells were infected by pRS or pRS/AR and drug-selected for 3 days. Next, cellular extracts were prepared and analyzed by immunoblotting for AR expression. $\beta$-Actin was used as loading control. (e) LNCaP or LNCaP/myc cells were infected by pRS or pRS/AR and drug-selected. Ten days after seeding 500,000 cells, crystal violet staining was performed and relative cell numbers were calculated.

The fluorescence intensity of this dye decreases twofold with each cell division, thus allowing determination of the number of cell divisions. Almost all c-myc-expressing cells divided one to four times in 9 days, whereas almost no control cells divided during the same period of time (Figure 1d). These data further indicate that AR independence is acquired by a bulk population of c-myc-expressing cells without need of additional genetic events.

To address whether c-myc expression maintains the LNCaP tumorigenic phenotype in the absence of androgen, we performed soft agar assays. LNCaP/GFP and $\mathrm{LNCaP} /$ myc cells were plated in soft agar with or without androgen. As expected, control and c-myc-expressing cells grew in the presence of androgen (Figure 1e). By contrast, only c-myc-expressing cells were able to form colonies in the absence of androgen (Figure 1e). Together, these data demonstrate that c-myc can rescue LNCaP cells from growth arrest and loss of tumorigenic potential induced by AR inhibition.

Enhancement of AR signalling has been proposed as a mechanism contributing to $\operatorname{AIPC}(2,3)$. To assess whether c-myc bypasses androgen dependency through an increase in AR signalling, we analyzed expression of PSA, a well-characterized AR-upregulated gene (16), and PSMA, an AR-downregulated gene (17). Expression of cmyc did not increase the PSA level in either the presence or absence of bicalutamide (Figure 2a). In fact, ectopic cmyc expression reduced the PSA level (Figure 2a), and it further decreased after AR antagonist treatment (Figure 2a). Transactivation assays showed that c-myc could downregulate PSA promoter (data not shown), thus explaining the previous observation. In addition, c-myc expression had no effect on the upregulation of PSMA observed during AR inhibition (Figure 2a). After analyzing the effect of c-myc on AR activity at a molecular level, we examined an eventual crosstalk at a functional level. Indeed, as AR activity is known to inhibit neuroendocrine differentiation (18-20), we examined the ability of c-myc to inhibit neuroendocrine differentiation during antiandrogen treatment. c-myc had no effect on the acquisition of the neuron-like morphology (Figure 2b) and the increase of a neuroendocrine differentiation marker, neuron-specific enolase (Figure 2c), induced by AR antagonist treatment in LNCaP cells. These data strongly suggest that c-myc induced AR-independent growth independently of AR signalling.

To further confirm whether c-myc acted independently of AR signalling, we used RNA interference against AR in both control and c-myc-expressing cells. Cells infected with a $\mathrm{pRS} / \mathrm{AR}$ vector (see Methods) displayed reduced AR expression compared with cells infected with $p R S$ vector (Figure 2d). As expected, control LNCaP cells, in which AR expression had been silenced by pRS/AR, ceased growing (Figure 2e). By contrast, c-myc-expressing cells continued to grow despite low AR levels (Figure 2e), suggesting that ectopic c-myc expression maintained growth independently of AR. Moreover, although c-myc did not act through AR signalling, AR was still expressed in these cells at a normal level (Figure 2d).

As AR inhibition caused a decrease of c-myc protein levels (Figure 2a), we further analyzed whether c-myc was regulated by androgen. We first checked the effects of bicalutamide treatment on c-myc RNA and protein levels. AR inhibition resulted in a decrease of the c-myc 
protein level but did not affect the c-myc RNA level (Figure 3a), indicating that AR regulated c-myc at a posttranscriptional level. Furthermore, treatment of androgen-depleted cells with androgen reinduced c-myc expression (Figure 3b).

As AR regulated the c-myc level and c-myc overexpression bypassed androgen dependency, we investigated whether c-myc was required for the androgendependent growth of LNCaP cells. To address this question, we reduced c-myc expression with a stable RNA interference construct. Infection of LNCaP cells with the pRS/myc vector reduced c-myc levels (Figure $3 c$ ) and severely inhibited cell growth (Figure $3 \mathrm{~d}$ ).

To define which c-myc growth controlled pathways might be required for bypassing androgen dependency, we analyzed the expression of growth-related genes regulated by c-myc. Confirming the results described above, the c-myc level decreased during AR inhibition and was sustained when overexpressed (Figure 3e). We first exam- ined the RB and p27 pathways, as c-myc can overcome growth arrest induced by these pathways $(21,22)$. p16 accumulated during bicalutamide treatment and was associated with subsequent hypophosphorylation of RB and a decrease of E2F1 targets (E2F1 and cyclin A) in control cells (Figure 3e). In contrast, although the level of p16 was constitutively high in c-myc-expressing cells, RB remained hyperphosphorylated during bicalutamide treatment, and levels of E2F1 and cyclin A remained high (Figure 3e). The level of Skp2 protein, a ubiquitin ligase responsible for $\mathrm{p} 27$ degradation (23), decreased during $\mathrm{AR}$ inhibition and was also maintained in c-mycexpressing cells. As expected, the inverse level of expression was observed with p27 (Figure 3e). Furthermore, levels of CDK4, cyclin D1, hTERT, and ornithine decarboxylase, which are known to exert positive growth effects and to be regulated by c-myc $(14,24-26)$, were downregulated in control cells after bicalutamide treatment and sustained in $\mathrm{LNCaP} /$ myc cells (Figure 3e).

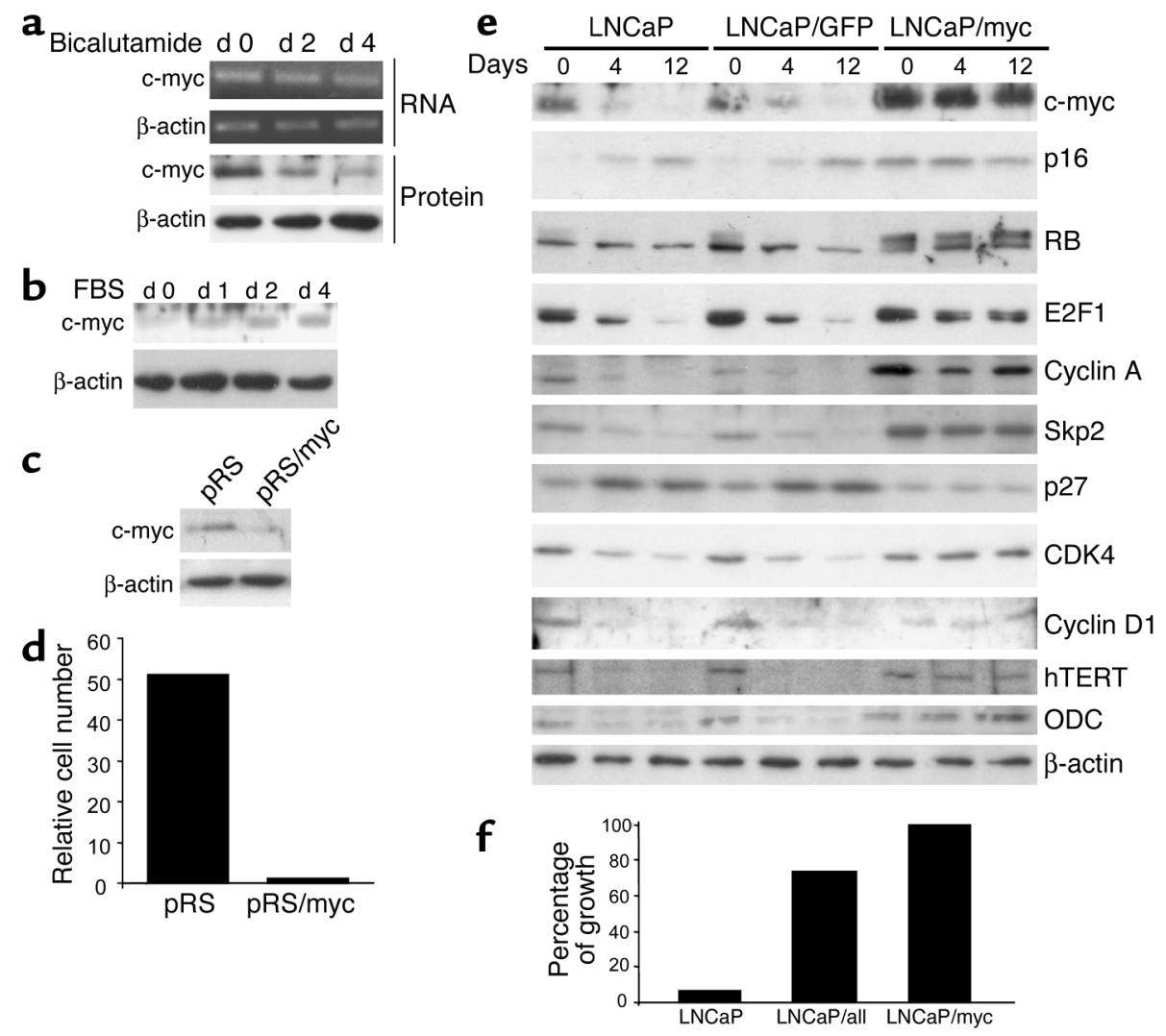

Figure 3

c-myc is an essential downstream growth effector of AR. (a) c-myc expression during AR inhibition. Media containing bicalutamide was added to inhibit AR activity for the indicated times. RNA and protein extracts were prepared and analyzed by RT-PCR and Western blot, respectively. (b) c-myc expression during AR activation. Cells were maintained in media with CDS and bicalutamide for 2 days. Next, normal media was added to restimulate AR activity for the indicated times. Cellular extracts were prepared and analyzed. (c) Silencing of c-myc expression. Cells were infected and selected for 2 days, then cellular extracts were prepared and analyzed for c-myc and $\beta$-actin expression. (d) Colony-formation assays. Cells $(400,000)$ were infected with pRS and pRS/myc retroviral vectors, and after 11 days the cells were stained with crystal violet. (e) Expression of growth regulators during AR inhibition. Cellular extracts were prepared before bicalutamide treatment or after 4 days and 12 days of treatment, resolved by SDS-PAGE, and analyzed by immunoblotting against c-myc, p16, RB, E2F1, cyclin A, Skp2, p27, CDK4, cyclin D1, hTERT, ornithine decarboxylase (ODC), and $\beta$-actin as a loading control. (f) Growth curve assays. Cells were infected successively with the different retroviruses and treated with polyamine. The growth curves were performed as described above. Shown is percentage of growth compared with $100 \%$ for c-myc-expressing cells treated with bicalutamide at day 12 . 


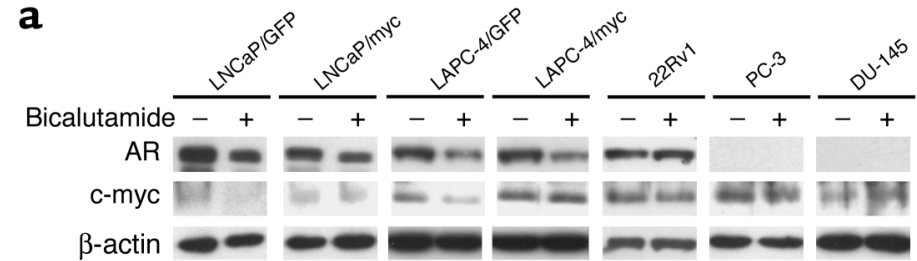

b
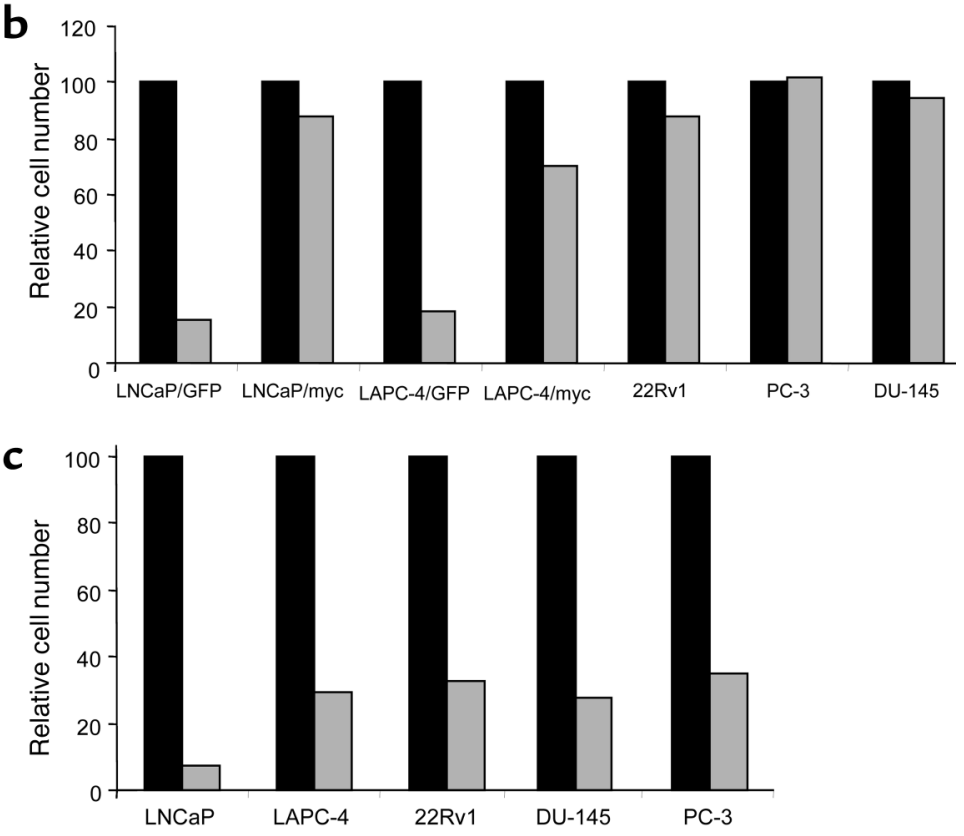

Thus levels of each of the c-myc targets tested were altered following bicalutamide treatment and this alteration was prevented by ectopic c-myc expression.

Accordingly, we assessed the effect of restoration of different c-myc-regulated pathways over growth in antiandrogen-treated cells. LNCaP cells were successively infected by retroviral constructions (E7, Skp2, hTERT, CDK4, cyclin D1) or treated with spermidine (a downstream active polyamine). Restoration of individual pathways or several combinations of these pathways resulted, respectively, in a minimum to a progressive overcoming of antiandrogenic effects (data not shown). Simultaneous manipulation of all these pathways allowed LNCaP cells to overcome androgen dependence to about $75 \%$ of that observed by c-myc expression (Figure 3f), suggesting that the c-myc effect involved additional pathways. These results also suggest that the c-myc effect was not exerted through a single main pathway but through multiple pathways, in agreement with previous data $(25,27)$.

We have demonstrated that c-myc is a downstream target of AR required for AR-dependent and AR-independent growth, and is sufficient, when constitutively expressed, for both androgen- and AR-independent growth in LNCaP cells. To confirm these results, we examined c-myc protein regulation in one additional androgen-dependent cell line (LAPC-4) and three human androgen-independent cancer cell lines (22Rv1, PC-3, and DU-145). As expected, c-myc expression was not sensitive to AR antagonist treatment in androgen-

\section{Figure 4}

c-myc is required for the growth of human AIPC cells. (a) Cells were treated with or without bicalutamide for 5 days. Cellular extracts were prepared, resolved by SDSPAGE, and analyzed by immunoblotting against c-myc, $A R$, and $\beta$-actin as a loading control. (b) The cells were seeded at low density and treated with (gray bars) or without (black bars) bicalutamide. After 2 weeks of treatment for LAPC- 4 cells or 10 days for the other cell lines, crystal violet staining was performed and relative cell numbers were calculated and normalized to $100 \%$ for untreated cells. (c) Cells were seeded at low density and infected with pRS (black bars) or pRS/myc (gray bars). After drug selection, the cells were split 1 to 8 and allowed to grow for 1-2 weeks. Crystal violet staining was performed and relative cell numbers were calculated and normalized to $100 \%$ for untreated cells.

independent, AR-negative cancer cell lines (PC-3 and DU-145) (Figure 4a). In androgenindependent, AR-positive 22Rv1 cells, AR antagonist treatment did not decrease c-myc levels, in contrast to androgen-dependent, AR-positive, LNCaP and (to a lesser extent) LAPC-4 cells (Figure 4a). Overexpression of c-myc in LAPC-4 cells was able to overcome growth arrest induced by AR antagonist treatment as it did in LNCaP cells (Figure 4b). The AIPC cells, which constitutively expressed c-myc, showed similar growth characteristics in the presence or absence of AR antagonist (Figure 4b). Thus, according to our results, c-myc should be essential for prostate cancer cell growth (AR-dependent or -independent). To address this point we looked at the growth of all these human prostate cancer cell lines when the c-myc level was decreased by RNA interference experiments. As predicted, RNA interference directed against c-myc resulted in growth arrest of both androgen-dependent and -independent cell lines (Figure 4c).

Chemotherapy, although currently not very efficient, is the major treatment for AIPC (1). As an intact p53 pathway is potentially required for effective action of DNA-damaging drugs such as etoposide, we assessed the integrity of this pathway in c-myc-overexpressing cells. In control and c-myc-expressing androgen-dependent (LNCaP) and androgen-independent (22Rv1) cells, etoposide treatment stabilized p53 and induced both p21 and GADD45 targets (Figure 5a), thus proving that the p53 pathway is not altered by c-myc expression. Next, we analyzed the growth effect of antiandrogen and/or etoposide treatment in $\mathrm{LNCaP}$ cells expressing c-myc and/or p53DN. Growth of both control and c-mycexpressing cells was inhibited by etoposide (Figure $5 \mathrm{~b}$ ). Expression of p53DN rendered c-myc-expressing cells and control cells resistant to etoposide treatment (Figure $5 \mathrm{~b}$ ). However, although inactivation of only the p53 pathway was not sufficient to bypass androgen dependency (Figure 5c), the combined expression of both c-myc and p53DN rendered cells resistant to both bicalutamide and etoposide (Figure 5d). Thus, the androgen- 


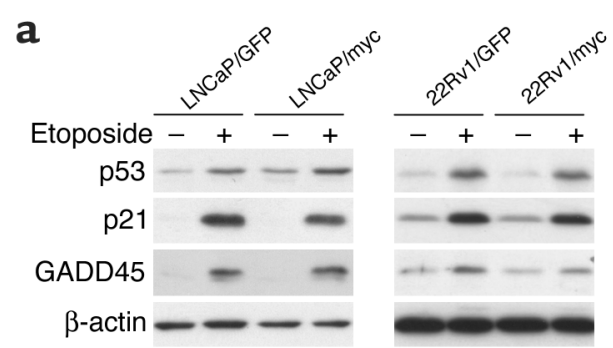

b

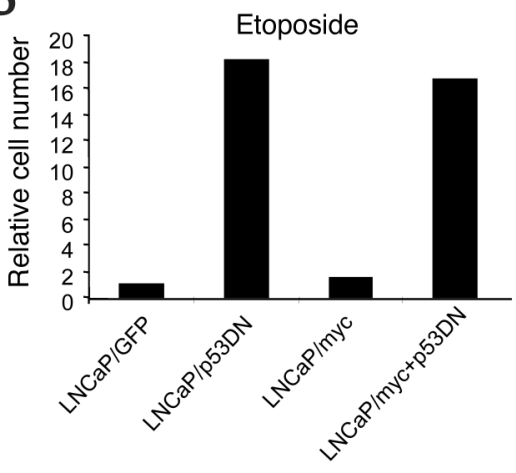

c

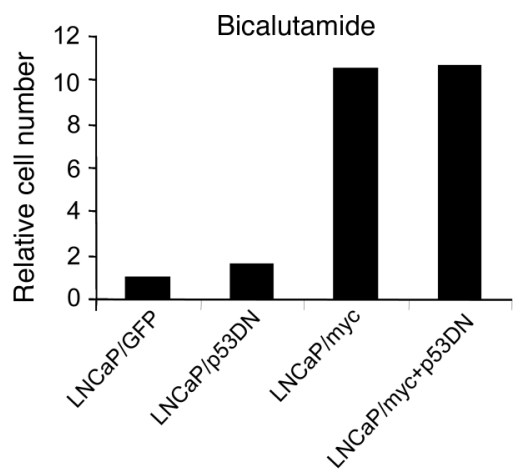

d

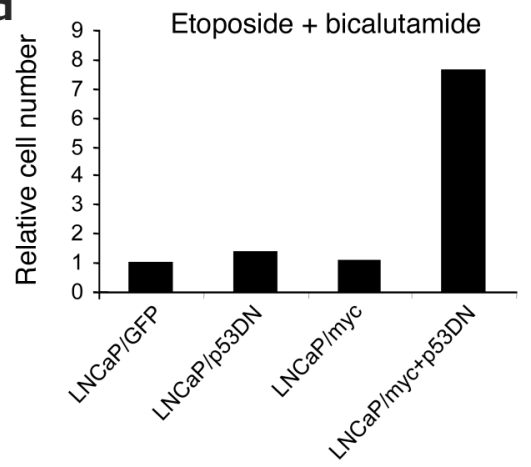

Figure 5

c-myc cells are sensitive to the p53 pathway. (a) One million infected cells were seeded in $10-\mathrm{cm}$ dishes. One day later, they were treated overnight with or without $25 \mu \mathrm{M}$ (LNCaP) or $50 \mu \mathrm{M}$ (22Rv1) etoposide. Cellular extracts were prepared, resolved, and transferred before analysis for p53, p21, and GADD45 expression. $\beta$-Actin was used as a loading control. (b) After infection, 750,000 cells were seeded in $10-\mathrm{cm}$ dishes and treated with $2 \mu \mathrm{M}$ etoposide at day 1 and day 7 . The cells were stained with crystal violet at day 17 , and relative cell numbers were calculated. (c) After infection, 250,000 LNCaP cells were seeded, treated with bicalutamide for 15 days, stained with crystal violet, and analyzed. (d) After infection, 750,000 cells were seeded in $10-\mathrm{cm}$ dishes and treated with bicalutamide every 2 days and with etoposide at day 1 and day 7 . The cells were stained with crystal violet at day 19 and analyzed. independent c-myc-expressing cells retain a functional p53 pathway and sensitivity to the chemotherapeutic drug etoposide, suggesting that c-myc itself is not involved in chemotherapeutic resistance.

\section{Discussion}

Prostate cancer is one of the most commonly occurring and mortal cancers in western countries. Proliferation of prostate tumors is dependent of AR, and hence androgen ablation is a central therapeutic approach. Despite use of this therapy, an androgen-refractory status almost invariably develops with time, with an eventually fatal outcome. Understanding molecular mechanisms triggered by AR signalling and more generally involved in AIPC development remains critical for the basic understanding of prostate cancer biology as well as for designing new potential targets during AIPC development and progression.

In this study, we show that overexpression of c-myc is sufficient to induce androgen-independent growth of androgen-dependent cells. Previous reports have suggested a potential role for c-myc in the development of androgen-refractory disease because of the high frequency (up to $70 \%$ ) of c-myc amplification in AIPC (10, 12). In addition, inactivation of c-myc inhibitors BIN1 (28) and MXI1 (29) is also observed in prostate cancer. Our data suggest that c-myc does not act through an increase of AR activity because c-myc did not increase PSA expression $(3,30)$ and did not decrease PSMA expression (17). Functional data further confirm these results as neuroendocrine differentiation induced by AR inhibition (18-20) was not overcome by c-myc expression, and more importantly, AR silencing in c-myc-expressing cells did not prevent cell growth.

Our results suggest that c-myc is a downstream target of AR, as the c-myc protein level is regulated by AR activity and as c-myc is required for androgen-dependent growth. The mechanism of c-myc regulation by AR is unclear, but our data suggest that c-myc is regulated at a posttranscriptional level since mRNA levels remained unchanged. It has been suggested in the literature that there is a shift from an inhibitory effect of AR on c-myc expression in cells in which AR induces differentiation $(31,32)$ to an activator effect in cells in which $\mathrm{AR}$ induces proliferation $(33,34)$.

We have also demonstrated that c-myc expression could immortalize human primary prostate epithelial cells by inhibiting the $\mathrm{p} 16-\mathrm{RB}$ pathway and inducing hTERT expression (Gil et al., unpublished observations). The fact that c-myc can be activated by AR activity as shown in this and other studies $(33,34)$ supports a role for c-myc in the early stages of prostate cancer. Accordingly, it was demonstrated that directed c-myc expression in the prostate induces development of a prostatic intraepithelial neoplasia in the mouse (35).

Thus, we propose a model in which c-myc is regulated by $A R$ and required for AR-dependent growth. In this model, and according to our data, c-myc is needed for ARinduced proliferation because it controls the activity of major growth related proteins. Consequently, without AR activity, but with constitutive expression of c-myc, the cells grow without a requirement for AR signalling, even if AR expression can be maintained. The fact that c-myc is expressed in and required for androgen-independent 
human cancer cell growth, as shown here in 22Rv1, PC-3, and DU-145 cells, supports this model.

As previously stated, the main hypotheses on development of AIPC rely on sustained AR activity during antiandrogen treatment resulting from either appearance of AR mutants, amplification of $A R$, or an increase in AR signalling (2). According to our data, AR signalling is sustained in c-myc-expressing cells. In the literature, numerous reports have shown that c-myc (10, $12)$ or $A R(36,37)$ can be amplified, and at least one report has shown that both genes can be coamplified in AIPC (38). Inactivation of $A R$ in prostate cancer has been reported in rare cases (39). Moreover, the development of recurrent tumors without elevation of PSA levels and with a marked neuroendocrine differentiation after antiandrogen treatment has been reported (40), suggesting that recurrence occurred independently of $\mathrm{AR}$ signalling. In both cases, the c-myc expression status is unknown, but c-myc appears to be a candidate of choice, as we have shown that c-myc expression in vitro allowed growth of differentiated neuroendocrine cells during antiandrogen treatment.

In conclusion, we demonstrated that beside the classical hypotheses of increase of AR signalling or establishment of a bypass pathway (2), c-myc may induce androgen-independent growth through a downstream pathway. Indeed, we have shown that c-myc is regulated by $A R$ and is required for AR-dependent as well as -independent growth, suggesting that c-myc may be involved in development of AIPC, including that resulting from an increase of AR signalling.

\section{Acknowledgments}

We thank P. Kerai, A. Carnero, D. Monte, and R. Kypta for helpful suggestions and discussion of the manuscript, L. Martinez for technical assistance, and M. Pagano for the LZRS/Skp2 retroviral vector. This investigation was supported by a grant from Cancer Research UK. Jesús Gil is a recipient of long-term fellowships from the European Molecular Biology Organization (EMBO) and the Human Frontier Science Program.

1. Denmeade, S.R., and Isaacs, J.T. 2002. A history of prostate cancer treatment. Nat. Rev.Cancer. 2:389-396.

2. Feldman, B.J., and Feldman, D. 2001. The development of androgen-independent prostate cancer. Nat. Rev. Cancer. 1:34-45.

3. Craft, N., Shostak, Y., Carey, M., and Sawyers, C.L. 1999. A mechanism for hormone-independent prostate cancer through modulation of androgen receptor signaling by the HER-2/neu tyrosine kinase. Nat. Med. 5:280-285.

4. Taplin, M.E., et al. 1999. Selection for androgen receptor mutations in prostate cancers treated with androgen antagonist. Cancer Res. 59:2511-2515.

5. Zhao, X.Y., et al. 2000. Glucocorticoids can promote androgen-independent growth of prostate cancer cells through a mutated androgen receptor. Nat. Med. 6:703-706.

6. Edwards, J., et al. 2001. Amplification of the androgen receptor may not explain the development of androgen-independent prostate cancer. BJU Int. 88:633-637.

7. Marcelli, M., et al. 2000. Androgen receptor mutations in prostate cancer. Cancer Res. 60:944-949.

8. Savinainen, K.J., et al. 2002. Expression and gene copy number analysis of ERBB2 oncogene in prostate cancer. Am. J. Pathol. 160:339-345.

9. Signoretti, S., et al. 2000. Her-2-neu expression and progression toward androgen independence in human prostate cancer. J. Natl. Cancer Inst. 92:1918-1925.

10. Nupponen, N.N., Kakkola, L., Koivisto, P., and Visakorpi, T. 1998. Genetic alterations in hormone-refractory recurrent prostate carcinomas. Am. J. Pathol. 153:141-148.

11. Visakorpi, T., et al. 1995. Genetic changes in primary and recurrent prostate cancer by comparative genomic hybridization. Cancer Res. 55:342-347.

12. Kaltz-Wittmer, C., et al. 2000. FISH analysis of gene aberrations (MYC, CCND1, ERBB2, RB, and AR) in advanced prostatic carcinomas before and after androgen deprivation therapy. Lab. Invest. 80:1455-1464.

13. Nesbit, C.E., Tersak, J.M., and Prochownik, E.V. 1999. MYC oncogenes and human neoplastic disease. Oncogene. 18:3004-3016.

14. Wang, J., Xie, L.Y., Allan, S., Beach, D., and Hannon, G.J. 1998. Myc activates telomerase. Genes Dev. 12:1769-1774.

15. Kasibhatla, S., et al. 1998. DNA damaging agents induce expression of Fas ligand and subsequent apoptosis in T lymphocytes via the activation of NF-kappa B and AP-1. Mol. Cell. 1:543-551.

16. Wolf, D.A., Schulz, P., and Fittler, F. 1992. Transcriptional regulation of prostate kallikrein-like genes by androgen. Mol. Endocrinol. 6:753-762.

17. Wright, G.L., Jr., et al. 1996. Upregulation of prostate-specific membrane antigen after androgen-deprivation therapy. Urology. 48:326-334.

18. Ahlgren, G., et al. 2000. Regressive changes and neuroendocrine differentiation in prostate cancer after neoadjuvant hormonal treatment. Prostate. 42:274-279.

19. Ismail, A.H., Landry, F., Aprikian, A.G., and Chevalier, S. 2002. Androgen ablation promotes neuroendocrine cell differentiation in dog and human prostate. Prostate. 51:117-125.

20. Wright, M.E., Tsai, M.J., and Aebersold, R. 2003. Androgen receptor represses the neuroendocrine transdifferentiation process in prostate cancer cells. Mol. Endocrinol. 17:1726-1737.

21. Goodrich, D.W., and Lee, W.H. 1992. Abrogation by c-myc of G1 phase arrest induced by RB protein but not by $\mathrm{p} 53$. Nature. 360:177-179.

22. Vlach, J., Hennecke, S., Alevizopoulos, K., Conti, D., and Amati, B. 1996 Growth arrest by the cyclin-dependent kinase inhibitor p27Kip1 is abrogated by c-Myc. EMBO J. 15:6595-6604.

23. Carrano, A.C., Eytan, E., Hershko, A., and Pagano, M. 1999. SKP2 is required for ubiquitin-mediated degradation of the CDK inhibitor p27. Nat. Cell Biol. 1:193-199.

24. Hermeking, H., et al. 2000. Identification of CDK4 as a target of c-MYC. Proc. Natl. Acad. Sci. U. S. A. 97:2229-2234.

25. Mateyak, M.K., Obaya, A.J., and Sedivy,J.M. 1999. c-Myc regulates cyclin DCdk4 and -Cdk6 activity but affects cell cycle progression at multiple independent points. Mol. Cell. Biol. 19:4672-4683.

26. Bello-Fernandez, C., Packham, G., and Cleveland, J.L. 1993. The ornithine decarboxylase gene is a transcriptional target of c-Myc. Proc. Natl. Acad. Sci. U. S. A. 90:7804-7808.

27. Berns, K., Hijmans, E.M., Koh, E., Daley, G.Q., and Bernards, R. 2000. A genetic screen to identify genes that rescue the slow growth phenotype of c-myc null fibroblasts. Oncogene. 19:3330-3334.

28. Ge, K., et al. 2000. Loss of heterozygosity and tumor suppressor activity of Bin 1 in prostate carcinoma. Int. J. Cancer. 86:155-161.

29. Eagle, L.R., et al. 1995. Mutation of the MXI1 gene in prostate cancer. Nat. Genet. 9:249-255.

30. Yeh, S., et al. 1999. From HER2/Neu signal cascade to androgen receptor and its coactivators: a novel pathway by induction of androgen target genes through MAP kinase in prostate cancer cells. Proc. Natl. Acad. Sci. U. S. A. 96:5458-5463.

31. Quarmby, V.E., Beckman, W.C., Jr., Wilson, E.M., and French, F.S. 1987. Androgen regulation of c-myc messenger ribonucleic acid levels in rat ventral prostate. Mol. Endocrinol. 1:865-874.

32. Ling, M.T., Chan, K.W., and Choo, C.K. 2001. Androgen induces differentiation of a human papillomavirus $16 \mathrm{E} 6 / \mathrm{E} 7 \mathrm{immortalized}$ prostate epithelial cell line. J. Endocrinol. 170:287-296.

33. Asadi, F.K., and Sharifi, R. 1995. Effects of sex steroids on cell growth and $\mathrm{C}$-myc oncogene expression in LN-CaP and DU-145 prostatic carcinoma cell lines. Int. Urol. Nephrol. 27:67-80.

34. Silva, I.S., Morsch, D.M., Urnauer, L., and Spritzer, P.M. 2001. Androgeninduced cell growth and c-myc expression in human non-transformed epithelial prostatic cells in primary culture. Endocr. Res. 27:153-169.

35. Zhang, X., et al. 2000. Prostatic neoplasia in transgenic mice with prostatedirected overexpression of the c-myc oncoprotein. Prostate. 43:278-285.

36. Brown, R.S., et al. 2002. Amplification of the androgen receptor gene in bone metastases from hormone-refractory prostate cancer. J. Pathol. 198:237-244.

37. Linja, M.J., et al. 2001. Amplification and overexpression of androgen receptor gene in hormone-refractory prostate cancer. Cancer Res. 61:3550-3555.

38. Miyoshi, Y., et al. 2000. Fluorescence in situ hybridization evaluation of cmyc and androgen receptor gene amplification and chromosomal anomalies in prostate cancer in Japanese patients. Prostate. 43:225-232.

39. Sasaki, M., et al. 2002. Methylation and inactivation of estrogen, progesterone, and androgen receptors in prostate cancer. J. Natl. Cancer Inst. 94:384-390.

40. Miyoshi, Y., et al. 2001. Neuroendocrine differentiated small cell carcinoma presenting as recurrent prostate cancer after androgen deprivation therapy. BJU Int. 88:982-983. 